\title{
Ultrasound biomicroscopic measurement of anterior chamber angle in premature infants
}

\author{
Hiroshi Kobayashi, Junichii Kiryu, Kaori Kobayashi, Takehisa Kondo
}

Department of

Ophthalmology,

Amagasaki Hospital,

Amagasaki, Japan

H Kobayashi

Department of

Ophthalmology and

Visual Science,

Graduate School of

Medicine Kyoto

University, Kyoto,

Japan

H Kobayashi

J Kiryu

K Kobayashi

\section{Department of}

Ophthalmology, Kobe

City General Hospital,

Kobe, Japan

T Kondo

Correspondence to:

Hiroshi Kobayashi,

Department of

Ophthalmology,

Amagasaki Hospital, 1-1-1

Higashi-daimotsu-cho,

Amagasaki, 660, Japan.

Accepted for publication 10 March 1997

Table 1 Mean, standard deviation (SD), and range of newworn variables and anterior segment measurements in 39 neonates

\begin{tabular}{lll}
\hline Variables & Mean $(S D)$ & Range \\
\hline Gestational age (weeks) & $33.3(4.0)$ & $25-39$ \\
Postconceptional age (weeks) & $34.4(4.1)$ & $27-40$ \\
Birth weight $(\mathrm{g})$ & $2322(757)$ & $963-3370$ \\
ACD $(\mu \mathrm{m})$ & $1303(201)$ & $901-1663$ \\
TIA $($ degree) & $23.13(5.71)$ & $8.75-28.44$ \\
AOD250 $(\mu \mathrm{m})$ & $142(52)$ & $34-229$ \\
AOD500 $(\mu \mathrm{m})$ & $220(78)$ & $50-332$ \\
Iris thickness $(\mu \mathrm{m})$ & $247(35)$ & $188-306$ \\
\hline
\end{tabular}

$\mathrm{ACD}=$ anterior chamber depth; TIA $=$ trabecular-iris angle; $\mathrm{AOD} 250=$ angle opening distance at $250 \mu \mathrm{m}$ from scleral spur; AOD500 = angle opening distance at $500 \mu \mathrm{m}$ from scleral spur; iris thickness $=$ thickness at the thickest part of the iris. difficulties, we have developed a method for measuring the anterior segment of neonates and obtaining live images by use of ultrasound biomicroscopy and a muscle hook with topical anaesthesia.Ultrasound biomicroscopy allows one to make precise measurements of the anterior segment in order to detect disorders and tumours. ${ }^{11-19}$ Our objective was to measure prospectively, for the first time, anterior chamber depth, angle width, and iris thickness in preterm infants in order to develop normative values. Correlation of these variables with postconceptional age and birth weight, as well as with each other, was evaluated.

\section{Patients and methods}

PATIENTS

Thirty nine preterm neonates were selected for study within 2 weeks after birth (see Table 1). The study protocol and consent forms were approved by the human subjects committee of Amagasaki Hospital. Informed consent for each infant was obtained from at least one parent. A neonatologist determined the infants' gestational age based on the maternal obstetric history, results of prenatal ultrasound, and a modified Dubowitz score. ${ }^{20}$ We calculated postconceptional age by adding the actual age of the infant at the time of ocular measurement to gestational age in weeks. Disappearance of the anterior vascular capsule of the lens was not used to estimate gestational age. The major factor that influenced the decision to include a specific premature infant in this study was the general stability of the newborn as related to its ability to undergo ocular evaluation. Infants who were small for gestational age were excluded from the study, as were infants with malformations, genetic disorders, those suspected of intrauterine infections, retinopathy of prematurity greater than stage II, or respiratory distress requiring assisted ventilation. Postconceptional age was approximated to the nearest week for data analysis.

ULTRASOUND BIOMICROSCOPIC MEASUREMENTS Ophthalmic and ultrasound biomicroscopic measurements were made of the right eye of each infant. All subjects were measured as soon as they were stable enough to undergo an ocular examination.

An ultrasonic biomicroscope 840 (ZeissHumphrey, San Leandro, CA, USA) with a 50 $\mathrm{Hz}$ transducer and an ultrasound biomicroscope UX-02 (Rion, Tokyo, Japan) with a 30 $\mathrm{Hz}$ transducer were used. Each infant was placed in a supine position. A neonatal Bangarter speculum, rather than an eye cup, 

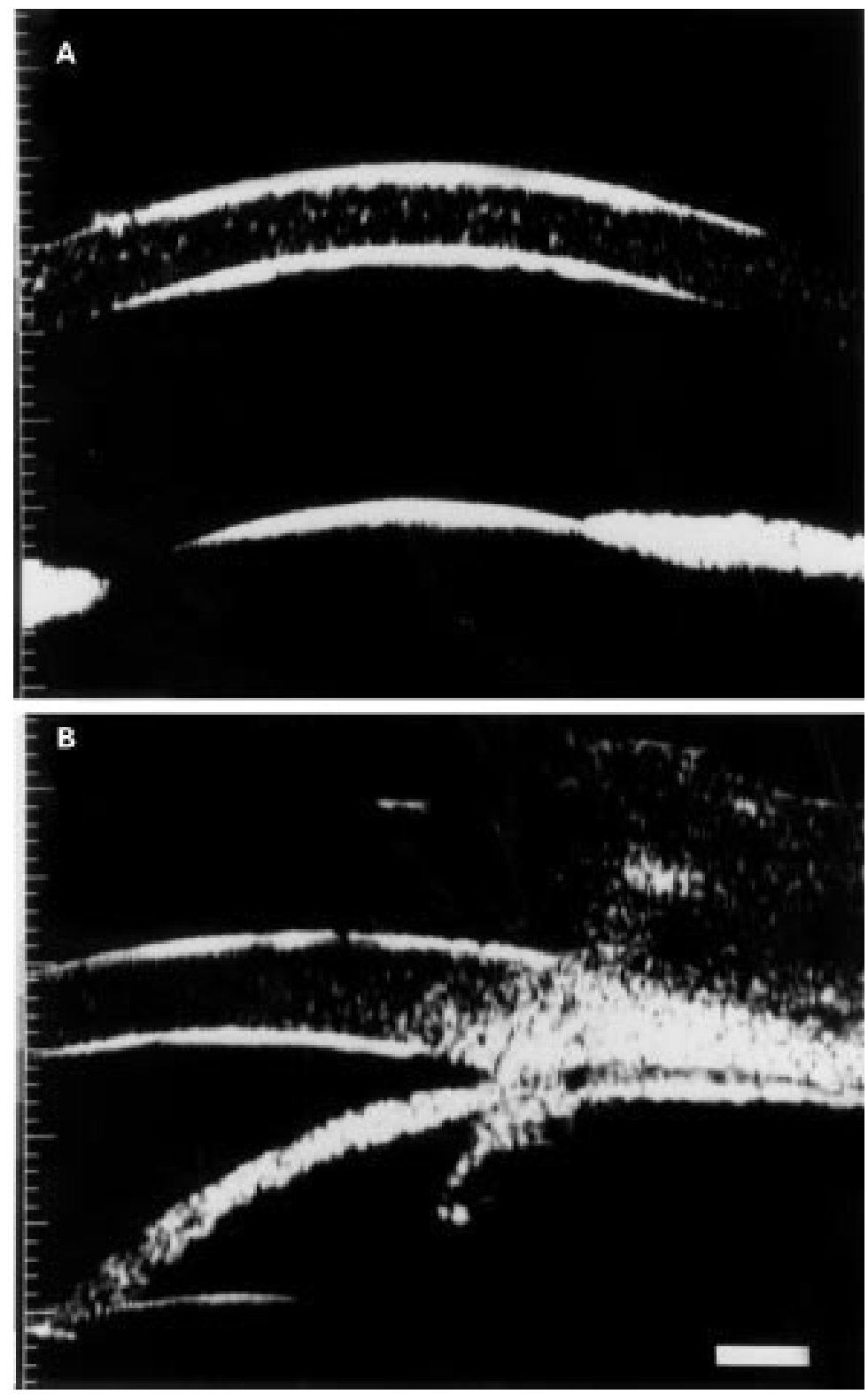

Figure 1 A $2640 \mathrm{~g}$ infant who was delivered by caesarean section at 38 weeks' gestation. (A) Ultrasound biomicroscopic image of the central anterior chamber. The ACD was 1320 $\mu m$. (B) Ultrasound biomicroscopic cross sectional view through the temporal angle region. The TIA, AOD250, AOD500, and thickness of the thickest part of the iris were $26.48^{\circ}$, $172 \mu \mathrm{m}, 216 \mu \mathrm{m}$, and $271 \mu \mathrm{m}$, respectively.

was used to separate the eyelids after a drop of $0.5 \%$ proxymetacaine (proparacaine) had been applied. The palpebral fissure was filled with $2.5 \%$ methylcellulose as a coupling medium. Scanning was performed by manipulating the eyeball with a muscle hook without pressure on the eyeball and placing the probe close to the area of interest. When a infant was not allowed out of an incubator, we examined through the ports of the incubator with the $30 \mathrm{~Hz}$ transducer. We repeated each measurement three times to confirm reproducibility and eliminate artefacts.

Anterior chamber depth was measured as an axial distance from the internal corneal surface to the lens surface. Ultrasound biomicroscopic cross sectional views through the angle region were obtained for most of the measurements, which were made in the temporal meridian; views were as vertical as possible as determined from the screen image. The following variables were measured: anterior chamber depth (ACD), trabecular-iris angle (TIA), angle opening distance at 250 and $500 \mu \mathrm{m}$ from the scleral spur (AOD250 and AOD500), and iris thickness, as described by Palvin et al. ${ }^{12}{ }^{13}$ Iris thickness was measured at the thickest part of the iris.

\section{STATISTICS}

Values were expressed as mean plus or minus the standard deviation and as a range. Unless otherwise specified, data were analysed by unpaired, unilateral $t$ tests. Stepwise regression analysis was used to evaluate which of the factors, age and weight, were predictive of anterior segment measurement. Regression analysis was performed to determine the precise relation of the ACD, TIA, AOD250, AOD500, and iris thickness to postconceptional age and birth weight. Because our interest is in the prediction of the relation for an individual infant, 95\% prediction limits were calculated for the eye measurements. These limits are wider than the $95 \%$ confidence limits for the mean eye measurement as given levels of predictor variables.

\section{Results}

Ultrasound biomicroscopic measurements of the anterior segment were obtained from 39 premature infants of 25 to 39 weeks' gestational age. The means, standard deviations, and ranges for gestational age, postconceptional age, and birth weight are shown in Table 1 . Birth weight showed a significant correlation with gestational age $(r=0.764, \mathrm{p}=$ 0.001). Efforts were made to examine the infants as soon as their medical conditions would allow. Age at examination ranged from 2 to 14 days (mean 6.6 (SD 4.1) days).

Figure 1 shows an ultrasound biomicroscopic image of the central anterior chamber and a cross sectional view through the temporal angle region of a $2640 \mathrm{~g}$ infant who was delivered at 38 weeks of gestation. The ACD, TIA, AOD250, AOD500, and thickness of the thickest part of the iris were $1320 \mu \mathrm{m}, 26.48^{\circ}$, $172 \mu \mathrm{m}, 216 \mu \mathrm{m}$, and $271 \mu \mathrm{m}$, respectively.

The ACD, TIA, AOD250, AOD500 and thickness of the thickest part of the iris for the group of entire infants are shown in Table 1. The ACD, TIA, AOD250, AOD500, and iris thickness significantly correlated with postconceptional age and birth weight (Figs 2, 3, and 4 , and Table 2). The slopes and intercepts for the regression analysis, with correlation coefficients with $\mathrm{p}$ values, are shown in Table 2 .

The ACD, TIA, AOD250, AOD500 and iris thickness significantly correlated to each other, as shown in Table 3.

\section{Discussion}

In this paper we have measured the anterior segments of premature infants and established normative values and $95 \%$ prediction limits for anterior chamber depth, trabecular-iris angle, 

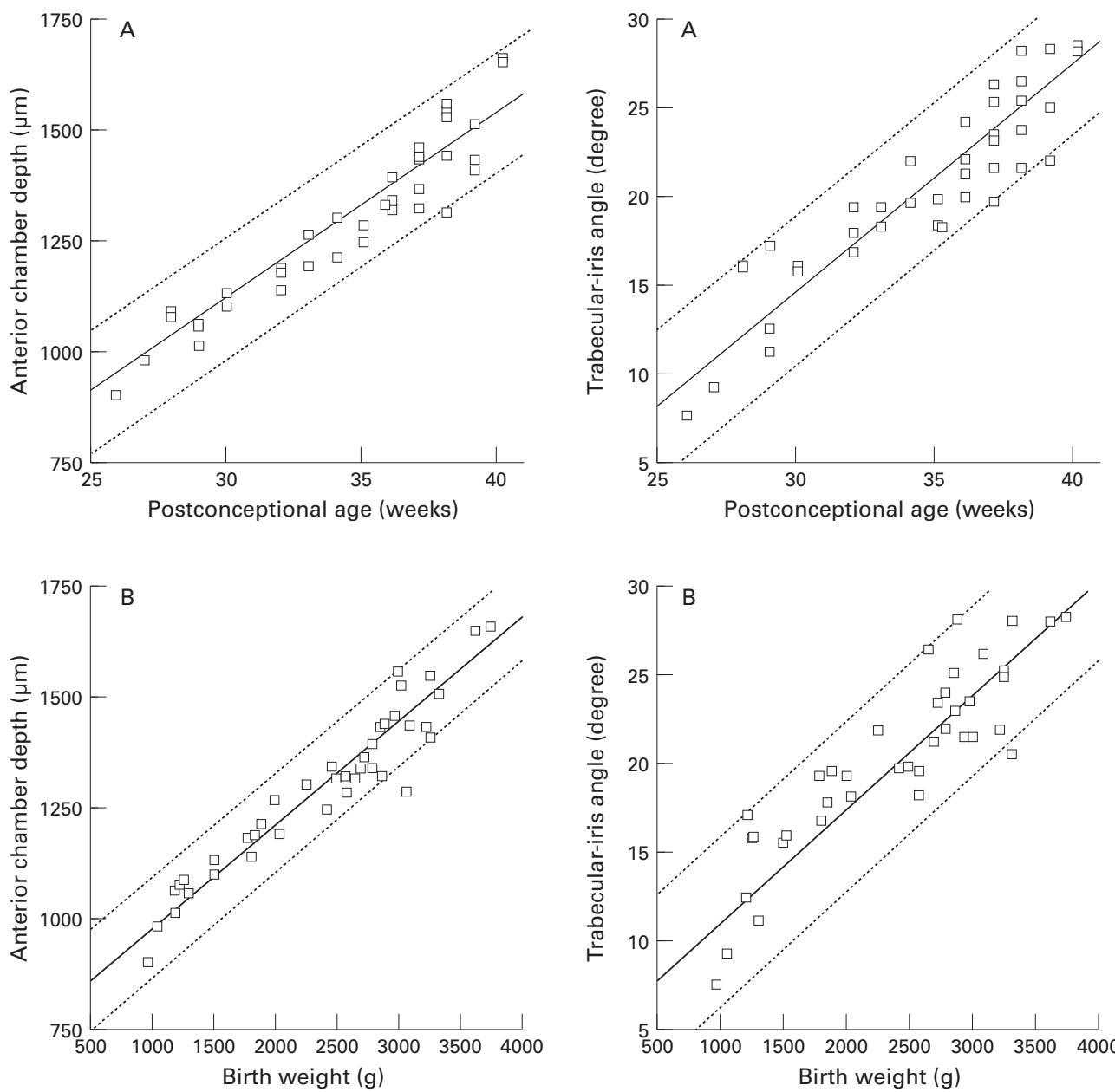

Figure 2 (A) Anterior chamber depth (ACD) in relation to postconceptional age with mean and $95 \%$ prediction limits $(r=0.962, p=0.0001 ; A C D=43.911 \times P C A-212.896)$. (B) ACD in relation to birth weight with mean and $95 \%$ prediction limits $(r=0.935, p=0.0001 ; A C D=0.231 \times$ $B W+761.709)$

AOD 250, AOD500 and iris thickness as related to postconceptional age and birth weight. We encountered no difficulty in obtaining ultrasound biomicroscopic images of the anterior segment in premature infants. Ultrasound biomicroscopy allows one to measure the anterior segments precisely even in the neonates. It is encouraging that distinct images of the exact area of interest can be obtained by a simple control of the eyeball with a muscle

Table 2 Regression analysis and correlation coefficients of anterior chamber depth, trabecular-iris angle, AOD250, AOD500, and iris thickness versus postconceptional age and birth weight

\begin{tabular}{llllrc}
\hline & $r$ & $r^{2}$ & $p$ Value & Slope & Intercept \\
\hline ACD $v$ PCA & 0.962 & 0.925 & 0.0001 & 43.911 & -212.896 \\
ACD $v$ BW & 0.935 & 0.874 & 0.0001 & 0.231 & 761.709 \\
TIA $v$ PCA & 0.892 & 0.796 & 0.0001 & 1.270 & -23.28 \\
TIA $v$ BW & 0.901 & 0.811 & 0.0001 & 0.007 & 5.20 \\
AOD250 $v$ PCA & 0.879 & 0.773 & 0.0001 & 11.822 & -261.873 \\
AOD250 $v$ BW & 0.886 & 0.785 & 0.0001 & 0.061 & 3.455 \\
AOD500 $v$ PCA & 0.806 & 0.650 & 0.0001 & 15.787 & -319 \\
AOD500 $v$ BW & 0.833 & 0.692 & 0.0001 & 0.083 & 30.394 \\
Iris thickness $v$ PCA & 0.709 & 0.503 & 0.001 & 5.071 & 76.174 \\
Iris thickness $v$ BW & 0.737 & 0.543 & 0.001 & 0.027 & 188.137 \\
\hline
\end{tabular}

PCA = postconceptional age; $\mathrm{BW}=$ birth weight $\mathrm{ACD}=$ anterior chamber depth; TIA = trabecular-iris angle; AOD250 $=$ angle opening distance at $250 \mu \mathrm{m}$ from scleral spur; AOD500 $=$ angle opening distance at $500 \mu \mathrm{m}$ from scleral spur; iris thickness $=$ thickness at the thickest part of the iris.

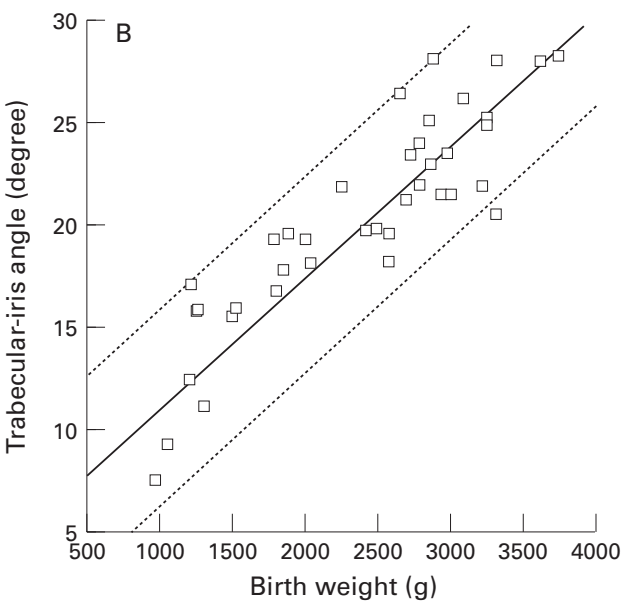

Figure 3 (A) Trabecular-iris angle (TIA) in relation to postconceptional age with mean and $95 \%$ prediction limits $(r=0.892, p=0.0001 ; T I A=1.270 \times P C A-23.28)$. (B) TIA in relation to birth weight with mean and $95 \%$ prediction limits $(r=0.901, p=0.0001 ; T I A=0.007 \times$ $B W+5.20)$.

hook. With the $30 \mathrm{~Hz}$ probe we were able to examine a neonate through the ports of an incubator. Even infants in poor medical condition can be examined in this way.

Only a few studies have attempted to determine morphometric values for preterm and term infants. There were studies of fetal cadaveric specimens. ${ }^{4}$ Postmortem movement of the lens makes necropsy studies less reliable than in vivo ultrasonographic studies for determining anterior chamber. ${ }^{21} 22$ Several investigators, using ultrasonography, have reported the anterior chamber depth of full term neonates. ${ }^{22-25}$ In all those studies, the central thickness of the cornea was included in the measurements. Our measurements showed that central corneal thickness ranged from 540 to $679 \mu \mathrm{m}$, with a mean of $620 \mu \mathrm{m}$ in preterm and term neonates (unpublished data). When data from our graphs are extrapolated at 40 weeks of gestation with the addition of the mean central thickness of the cornea, they correspond directly to Larsen's ultrasound measurements of the anterior chamber depth.

Anterior chamber depth, trabecular-iris angle, AOD250, AOD500, and iris thickness show a linear relation with postconceptional 

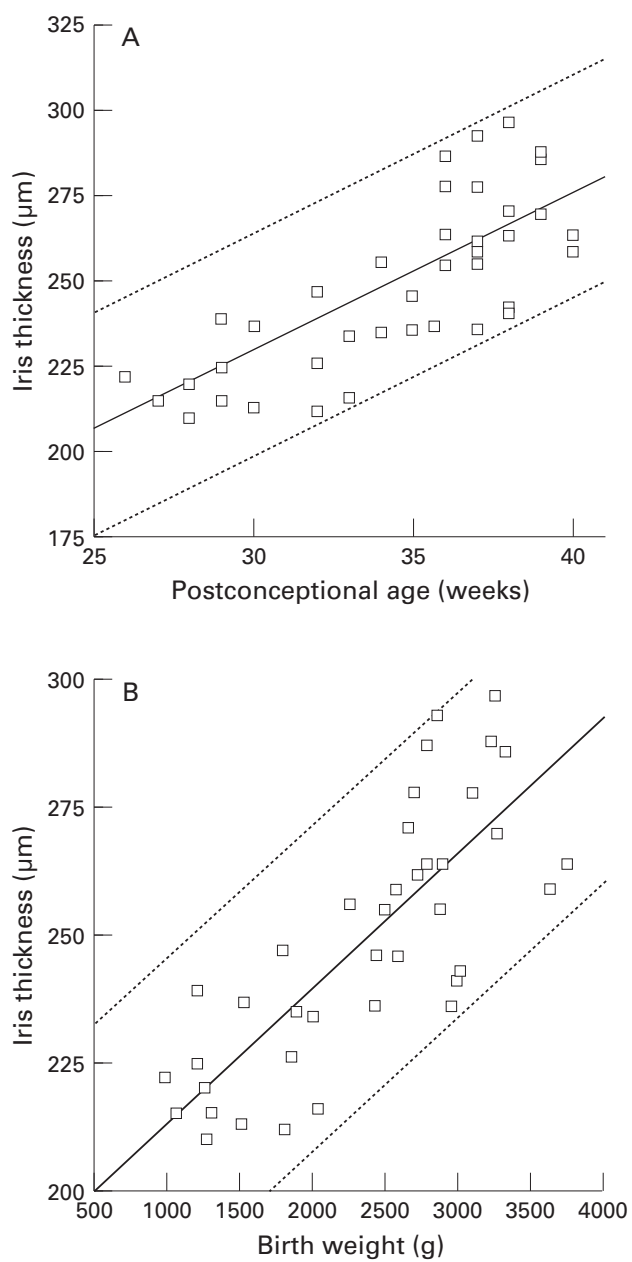

Figure 4 (A) Thickness of the thickest part of the iris in relation to postconceptional age with mean and $95 \%$ prediction limits $(r=0.709, p=0.001$; iris thickness $=$ $5.071 \times P C A+76.174)$. (B) Thickness of the thickest part of the iris in relation to birth weight with mean and $95 \%$ prediction limits $(r=0.737, p=0.001$; iris thicknes $=0.027 \times B W+188.137)$.

age and birth weight. Other ocular dimensions, including total axial length and corneal diameter, were correlated with postconceptional age and birth weight..$^{5-7} 910$ Musarella and Morin reported that corneal diameter correlated better with birth weight than with postconceptional age. ${ }^{7}$ Tucker et al found postconceptional age to be more significant predictive factor than birth weight. ${ }^{10}$ However, our results showed that anterior chamber depth, angle width, and iris thickness were all identically

Table 3 Regression analysis and correlation coefficients between anterior chamber depth, trabecular-iris angle, AOD250, AOD500, and iris thickness

\begin{tabular}{lllllr}
\hline & $r$ & $r^{2}$ & $p$ Value & Slope & Intercept \\
\hline ACD v TIA & 0.907 & 0.823 & 0.0001 & 29.92 & 686.67 \\
ACD v AOD250 & 0.908 & 0.825 & 0.0001 & 3.171 & 838.39 \\
ACD v AOD500 & 0.895 & 0.801 & 0.0001 & 2.147 & 817.367 \\
ACD v iris thickness & 0.660 & 0.436 & 0.0001 & 4.334 & 211.344 \\
TIA v AOD250 & 0.897 & 0.805 & 0.0001 & 0.095 & 6.660 \\
TIA v AOD500 & 0.893 & 0.797 & 0.0001 & 0.065 & 5.898 \\
TIA v iris thickness & 0.702 & 0.493 & 0.0001 & 0.140 & -14.616 \\
AOD250 v AOD500 & 0.902 & 0.814 & 0.0001 & 0.620 & 6.192 \\
AOD250 v iris thickness & 0.665 & 0.442 & 0.0001 & 1.25 & -168.604 \\
AOD500 v iris thickness & 0.572 & 0.327 & 0.0002 & 1.568 & -169.351 \\
\hline
\end{tabular}

$\mathrm{ACD}=$ anterior chamber depth; TIA $=$ trabecular-iris angle; $\mathrm{AOD} 250=$ angle opening distance at $250 \mu \mathrm{m}$ from scleral spur; AOD500 = angle opening distance at $500 \mu \mathrm{m}$ from scleral spur; iris thickness $=$ thickness at the thickest part of the iris. correlated with postconceptional age and birth weight. The greater statistical significance of our results may reflect the relatively large number of infants studied, as well as the accurate determination of postconceptional age.

The appearance of the anterior chamber angle in the neonate differs from that in adults. ${ }^{31}$ The scleral spur is closer to the apex of the angle in preterm and term infants than it is in adults. Interpretation of anatomical landmarks, including the scleral spur, can affect the measurement of ultrasound images. ${ }^{18}$ The scleral spur is easily detected even when the angle is poorly developed in premature infants. The scleral spur is an appropriate landmark for measuring the anterior chamber angle.

We established normative values for anterior segment in preterm newborns. These data provide the first information on the development of the anterior segment in immature eyes. However, we have not yet created growth curves for these variables, because we are following our patients longitudinally. The normative values should greatly aid ophthalmologists in evaluating anterior segment dimensions of premature neonates who are suspected of having congenital glaucoma and congenital anomalies.

1 Sampaolesi R, Caruso R. Ocular echometry in the diagnosis of congenital glaucoma. Arch Ophthalmol 1982;100:57477

2 Tarkkanen A, Uusitalo R, Mianowicz J. Ultrasonographic biometry in congenital glaucoma. Acta Ophthalmol 1983; 61:618-23.

3 Anderson DR. The development of the trabecular meshwork and its abnormality in primary infantile glaucoma. work and its abnormality in primary in
Trans Am Ophthal Soc 1981;79:458-85.

4 Sorsby A, Sheridan M. The eye at birth: measurements of the principle diameters in forty-eight cadavers. If Anat (London) 1960;94:192-7.

5 Harayama K, Amemiya T, Nishimura H. Development of the eyeball during fetal life. $\mathcal{F}$ Pediatr Ophthalmol Strabismus 1981;18:37-40.

6 Jeanty P, Dramaix-Wilmet M, Van Gansbeke D, Van Regemorter N, Rodesch F. Fetal ocular biometry by ultrasound. Radiology 1982;143:513-6.

7 Musarella MA, Morin JD. Anterior segment and intraocular pressure measurements of the unanesthetized premature infant. Metab Pediatr Sys Ophthalmol 1982;8:53-60.

8 Swan KC, Wilkins JH. Extraocular muscle surgery in infancy: anatomical factors. F Pediatr Ophthalmol Strabismus 1984;21:44-9.

9 Isenberg SJ, McCarty JW, Rich R. Growth of the conjunctival fornix and orbital margin in term and premature infants. Ophthalmology 1987;94:1276-80.

10 Tucker SM, Enzenauer RW, Levin AV, Morin JD, Hellmann J. Corneal diameter, axial length, and intraocular pressure in premature infants. Ophthalmology 1992;99:1296-300.

11 Pavlin CJ, Sherar MD, Foster FS. Subsurface ultrasound microscopic imaging of the intact eye. Ophthalmology 1990; 97:244-50.

12 Pavlin CJ, Harasiewicz K, Sherar MD, Foster FS. Clinical use of ultrasound biomicroscopy. Ophthalmology 1991;98: 287-95.

13 Pavlin CJ, Harasiewicz K, Foster FS. Ultrasound biomicroscopy of anterior segment structures in normal and glaucomatous eyes. Am f Ophthalmol 1992;113:381-9.

14 Pavlin CJ, McWhae JA, McGowan HD, Foster FS. Ultrasound biomicroscopy of anterior segment tumors. Ophthalmology 1992;99:1220-8.

15 Pavlin CJ, Ritch R, Foster FS. Ultrasound biomicroscopy in plateau iris syndrome. Am f Ophthalmol 1992;113:390-5.

16 Tello C, Chi T, Shepps G, Liebmann J, Ritch R. Ultrasound biomicroscopy in pseudophakic malignant glaucoma. Ophthalmology 1993;100:1330-4.

17 Potash SD, Tello C, Liebmann J, Ritch R. Ultrasound biomicroscopy in the pigment dispersion syndrome. Ophthalmology 1994;101:332-9.

18 Tello C, Liebmann J, Potash SD, Cohen H, Ritch R. Measurement of ultrasound biomicroscopy images: Intraobserver and interobserver reliability. Invest Ophthalmol Vis Sci 1994;35:3549-52.

19 Aslanides IM, Libre PE, Silverman RH, Reinstein DZ, Lazzaro DR, Rondeau MJ, et al. High frequency ultrasound imaging in pupillary block glaucoma. $\mathrm{Br} \mathcal{F}$ Ophthalmol 
20 Dubowitz LMS, Dubowitz V, Goldberg C. Clinical assessment of gestational age in the newborn infant. F Pediatr $1970 ; 77: 1-10$.

21 McCormick AQ. Transient phenomena of the newborn eye. In: Isenberg SJ, ed. The eye of infancy. St Louis: Mosby, 1994:67-72

22 Gernet H. Achsenlänge und Refraktion lebender Augen von Neugeborenen. Albrecht v Graefes Arch Ophthalmol 1964; 166:530-6.
23 Luyckx J. Mesure des composantes optiques de l'aeil du nouveauné par echographie ultrasonique. Arch Ophtal (Paris) 1966;26:159-70

24 Larsen JS. The sagittal growth of the eye. IV Ultrasonic measurement of the axial length of the eye from birth to puberty. Acta Ophthalmol 1971;49:873-86.

25 Blomdahl S. Ultrasonic measurements of the eye in the newborn infant. Acta Ophthalmol 1979;57:1048-56. 